\title{
Nature's sister journals in March
}

As a service to those readers of Nature who do not see its sister journals regularly, the following pages refer to some of the content of Nature Genetics, Nature Structural Biology and Nature Medicine.

\section{Continuing hunt for diabetes genes targets unique mini-satellites}

THE early-onset form of diabetes appears to be associated with a tandemly repetitive DNA sequence of variable overall length upstream of the insulin gene on human chromosome 11 (11p15.5). That conclusion, of interest in its own right, is reminiscent of how variable numbers of repetitive elements, usually, are associated with various inheritable neurological diseases, Huntington's disease, for example.

The role of the repeating elements in the genetics of early-onset diabetes (also known as "type I" and "insulin-dependent" diabetes, or "IDS") is outlined by two articles in the March issue of Nature Genetics. The new developments are the outcome of a decade-long search for the inheritable correlates of early-onset diabetes. No fewer than six genetic determinants of IDS have now been identified, of which the principal is a nature locus in the LA complex on chromosome 6 .

Linkage studies have previously located a susceptibility locus (called) to a $4.1 \mathrm{~KB}$ region of chromosome 11 that includes the insulin gene. A large group from the University of Oxford, including $\mathrm{S}$. T. Benett and J. A. Todd, together with collaborators from Norway, Denmark, Birmingham (England) and France, have now been able to show that coincides with the Variable Number Tandem Repeat (VENTURE) know for a decade to lie upstream of the start-point of the insulin gene (S. T. Benett et al. Nature Genet. 9, 284-292; 1995).

As mini-satellites go, this VENTURE is unusual in that the repeating element is 14 (sometimes 15) nucleotides long. A total of at least 11 variants of the sequence have been recognised, all of which are rich in guanine. Haploid chromosome sets may differ from each other in the number of repetitive units (from fewer than 40 to more than 150) and in the occurrence and order of variants of the basic repetitive sequence.

The proof that $V N T R$ alleles determine susceptibility to IDDM rests on studies of parental transmission to offspring (diabetic or otherwise) in 425 families from Britain, France and the United States, which has in turn been made possible by a successful technique for PCR fluores- cence-linked amplification of microsatellites, followed by electrophoresis with acrylamide and urea gel to give an estimate of the size of the VNTR allele.

One finding is that the shorter alleles are associated with susceptibility to earlyonset diabetes, but there is one ironical exception. The allele whose length is 698 base-pairs $(14 \times 50=700)$ appears to be protective; the irony is that this was the version of the mini-satellite first sequenced. The authors also conclude that most short alleles (but not that numbered 698) are associated with enhanced insulin expression. There is also evidence of maternal and paternal imprinting (differences of the efficacy of the differently sourced genes) in the diploid genome.

In an accompanying paper, Guilia Catignani Kennedy, Michael S. German and William J. Rutter from the University of California, San Francisco, show that the VNTR locus upstream from the insulin gene is capable of binding to molecules of the transcription factor known as Pur-1 (Nature Genet. 9, 293-298; 1995). Their objective is to study the effects of repetitive units of different sequence on the efficacy of gene promoter regions in which they are included. using a novel in vitro system in which pancreatic $\beta$-cells are used to assess the effects of various $V N T R$

Also in Nature Genetics, March: - The mouse gene Mash2, which encodes a transcription factor required for trophoblast development, is genomically imprinted (in favour of the maternal gene) and is located within a cluster of such genes on mouse chromosome 7.

- Foreign genes may be transferred to mouse embryos at any stage of gestation by injecting plasmid-lipopolyamine complexes into pregnant females.

- A point mutation of the gene for the copper-transporting protein ceruloplasmin has been demonstrated to be responsible for inherited systemic haemosiderosis, but it is not clear why neurological and other organ damage is caused by the deposition of iron, rather than copper, in affected tissues.

Imprinting of the Xist gene (on the X chromosome) appears to be linked with methylation. sequences within the promoter region on the expression of reporter genes. A complication for the further study of susceptibility to IDDM is the finding that there are, indeed, significant differences between the effects of different nucleotide sequences.

Kennedy et al. that some of their conclusions may be undermined by the finding of Bennett et al. that the "short" allele used in their study may be that found to counteract susceptibility to diabetes. They go on to make two other important points:

First, the location of the VNTR locus within the promoter region of the insulin gene does not, of itself, account for the aetiology of IDDM, which involves the auto-immune destruction of pancreatic _cells, but the excess of insulin production above some threshold may be the trigger for such a reaction.

Second, the VNTR locus is found, among vertebrates, only in primates, suggesting a relatively recent evolutionary origin for this mini-satellite and even raising questions about the function of this nucleotide complex other than to confer susceptibility to IDDM.

The same issue of Nature Genetics includes a report by a largely French group (based at two INSERM units at Paris) of a link between a point-mutation of the human glucagon receptor gene and the occurrence of "non-insulin dependent" diabetes (or NIDDM). The glucagon receptor is a transmembrane protein whose gene occupies more than $5.5 \mathrm{~kb}$ on human chromosome 17 , up against the "q" telomere, and is coupled cytomplasmically to G-protein.

J. Hager et al. (Nature Genet. 9, 299$304 ; 1995)$ describe a significantly greater incidence of this mutation among patients with NIDDM from France and Sardinia (where the overall incidence of NIDDM exceeds 10 per cent for those over 60 ). They also show that he affinity of glucagon for the mutated receptor is much reduced, compared with binding to the unmutated version.

Even so, the mutated glucagon receptor is not an unequivocal indicator of the occurrence of NIDDM; in the Sardinian population, this version of the gene is only three times more common in late-onset diabetics than in the general population. But, intriguingly, the effect of the mutation is to make the amino acid sequence of a highly conserved region of the glucagon receptor identical with that of the rat. 


\section{'Catalytic triad' modified}

A NEW structure of an esterase from the potato scab-inducing bacterium Streptomyces scabies (Y. Wei, et al. Nature struct. Biol. 2, 218-223) may compel a reassessment of how the so-called "catalytic triad" functions in enzymes generally. The $2.1 \AA$ structure has been obtained by Z. S. Derewanda's group at the University of Alberta with collaboration from the University of Minnesota and the Novo-Nordisk Research Laboratories at Copenhagen.

A detailed structure

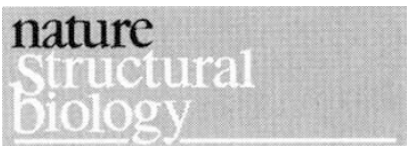

Proteasomes are cylindrical assemblages of twenty-eight proteins arranged as a stack of four seven-membered rings, together with a number of accessory proteins. They are present in both eukaryotes and prokaryotes, being responsible for the destruction of damaged and misfolded proteins as well as short-lived regulatory proteins marked for destruction by tagging with the protein ubiquitin.

Wenzel and Baumeister, from the MaxPlanck institute at Martinsried (Munich), have used proteasomes from the archaebacterium Thermoplasma acidophilum to show that only fully unfolded proteins are degraded. They of the chymotrypsin molecule a quarter of a century ago first drew attention to the involvement of three amino acids, from different parts of the molecular sequence, in the active site of the enzyme. Since then, the same catalytic triad, with variations, has been found in a range of enzymes all concerned with the breakdown of biological material.

There is often no other homology than three amino acids, a serine, a histidine and an acidic residue (usually either glycine or asparagine), brought together from different points in the protein sequence. The serine is the chemically reactive group, making a nucleophilic attack on the enzyme's substrate. In chymotrypsin the chemical reactivity was thought to be achieved by a 'proton-shunt' in which the histidine withdraws a hydrogen atom from the serine residue and itself loses a proton to the acidic aspartate.

\section{Proteasome function revealed}

Although there are many forms of the catalytic triad, it has hitherto been thought that the third acidic member is essential to stabilize the positively charged histidine. Now, in the esterase from $S$. scabies, a bulky aromatic tryptophan that cannot function as an acid turns out to be the third member of the triad. An analogous situation has been found in a catalytic antibody, in which a serine and a histidine appear to function as if in a catalytic triad without the equivalent of a third acidic member.

In the light of the new $S$. sabies esterase structure, the authors suggest that the role of the third member of the catalytic triad may not be electrochemical, but rather that of correctly orienting the histidine, in which case the term 'catalytic diad' may be more appropriate.

An elegant series of experiments has now thrown light on the way in which the cytoplasmic structures called proteasomes differentiate between proteins to be degraded by proteosomes and those left intact.(T. Wenzel \& W. Baumeister Nature struct. Biol. 2, 199-204; 1995). suggest that degradation takes place within the proteasome and that only proteins which can thread into its inner cavity are

Graphic evidence for that is provided by the observation that when a bulky gold particle was attached to the B-chain of

\section{Glycosylation of IgG and rheumatoid arthritis}

THE possibility that the activation of complement in the causation of inflammation in rheumatoid arthritis is brought about by abnormal glycosylation of IgG molecules is suggested by Rajneesh Mulhotra et al. and Raymond A.

Dwek, respectively from the MRC Immunochemistry Unit and the Glyocobiology Institute of the Biochemitry Department, University Oxford, in the March issue of Nature Medicine.

With collaborators, the two groups build on earlier observations that the glycosylation pattern at the Asn 297 site of IgG is abnormal in rheumatoid arthritis, with the loss of either one or two of the terminal galactose residues in a normally branched chain (R. Mulhotra et al. Nature Med. 1, 237-243; 1995). The authors now demonstrate that, in the absence of the degraded.

\section{Nature Medicine - other points}

A correspondent from the Queen's University, Belfast, pleads that optimism about the use of gene therapy for the treatment of cystic fibrosis (CF) should not conceal from physicians and their patients that CF is a systemic disease, affecting other organs than the lungs, and that there is a "need to help patients live with the reality of their disease". - The observation, during the use of adenovirus vectors for the delivery of human CFTR genes to bronchial tissue, of imflammation linked with the release of interleukin- 6 has led to the further observation that this cytokine is also produced in the inflammatory
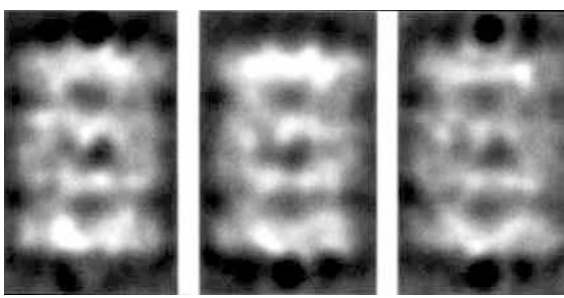

insulin, which is normally swiftly degraded, the peptide became resistant to degradation. Indeed, such a gold particle can be seen under the electron microscopy plugging the ends of the proteasomes (as in the figure).

This does not explain how the tagging of proteins with ubiquitin leads to their destruction, but the authors suggest that the accessory proteins at the ends of the proteasome, which were not present in this study, may act as 'reverse-chaperones' selectively unfolding proteins so that they can be fed into the cellular equivalent of a shredding machine.

terminal sugars, binding to mannose binding protein (MBP) is enhanced and that one consequence is the activation of complement. This chain of events is substantiated
both biochemically and by structural studies, which reveal a similarity of the structure of multimers of $\mathrm{MBP}$ with the structure of $\mathrm{Clq}$, the presumed initiator of the complement cascade, both of which include regions resembling collagen in structure.

The inference is that the presence of IgG antibodies lacking terminal galactose residues may cause the chromic inflammation of the synovium characteristic of rheutmatoid arthritis, but the authors raise the question whether imperfectly glycosylated $\operatorname{IgG}$ may have an earlier function in the causation of the disease. $\square$

lesions in the CF lung.

- On the assumption that the failure of grafts of fetal tissue in the treatment of rat models of Parkinson's disease may be a consequence of oxygen toxicity, transgenic cells from the substantia nigra designed to overexpress ( $\mathrm{Cu}-\mathrm{Zn})$-superoxide dismutase are reported to survive for longer.

- The finding in a small number of people with acute depression of antigens belonging to the Borna virus, originally recognized as responsible for neurological disease in horses, suggests a new line of enquiry for psychiatrists with an interest in PCR techniques. 